\title{
RANDOM MATRIX Generators FOR OptimiziNG A Fuzzy Biofuel Supply Chain System
}

\author{
Timothy GANESAN ${ }^{1}$, Pandian VASANT ${ }^{2, *}$, Pratik SANGHVI ${ }^{1}$, \\ Joshua THOMAS ${ }^{3}$, Igor LITVINCHEV ${ }^{4}$ \\ ${ }^{1}$ Royal Bank of Canada, Canada \\ ${ }^{2}$ Universiti Teknologi Petronas, Malaysia \\ ${ }^{3}$ UOW Malaysia, KDU Penang University College, Malaysia \\ ${ }^{4}$ Nuevo Leon State University, Mexico \\ *Corresponding Author:Pandian VASANT (Email: pvasant@gmail.com) \\ (Received: 12-Nov-2019; accepted: 3-Feb-2020; published: 31-Mar-2020) \\ DOI: http://dx.doi.org/10.25073/jaec.202041.268
}

\section{Introduction}

Abstract. Complex industrial systems often contain various uncertainties. Hence sophisticated fuzzy optimization (metaheuristics) techniques have become commonplace; and are currently indispensable for effective design, maintenance and operations of such systems. Unfortunately, such state-of-the-art techniques suffer several drawbacks when applied to largescale problems. In line of improving the performance of metaheuristics in those, this work proposes the fuzzy random matrix theory (RMT) as an add-on to the cuckoo search (CS) technique for solving the fuzzy large-scale multiobjective (MO) optimization problem; biofuel supply chain. The fuzzy biofuel supply chain problem accounts for uncertainties resulting from fluctuations in the annual electricity generation output of the biomass power plant [kWh/year]. The details of these investigations are presented and analyzed.

\section{Keywords}

Industrial optimization often involves systems containing various complexities and uncertainties - thus requiring heavy computational effort when performing optimization. In such scenarios metaheuristics play a prominent role (Ganesan et al.[25]; Ganesan et al.[26]; Yang [66]; Ganesan et al. [24]; Ganesan et al. [27]; Hong et al.[32]; Dong et al. [21]). Decision makers are globally facing various optimization challenges when optimizing supply chains - this is attributed to its large-scale and complex structure. Currently various state-of-the-art tools have been developed to overcome these challenges where they have been used to:

- Model these supply chains (Seuring [55]; Brandenburg et al. [12]; Ahi and Searcy [3])

- fficiently optimize the decision making process (Ogunbanwo et al. [47]; Mastrocinque et al. [43])

Fuel supply chains have broad applications span-

Random matrix theory, fuzzy framework, cuckoo search, biofuel supply chain, multiobjective (MO), large-scale optimization.

ning across diverse industrial sectors. For instance in Lin et al. [38], the annual biomassethanol production cost in a fuel supply chain was minimized. In that work, the large-scale 
supply chain model consisted of: stacking, in-field preprocessing, transportation, transportation, biomass harvesting, packing/storage, ethanol production and ethanol distribution. Aiming to reduce the cost of production in a biorefinery (to approximately 62\%), the researchers used the mixed integer programming technique. Another interesting work on a switchgrass-based bioethanol supply chain (located in North Dakota, U.S) was presented in the work of Zhang et al. [71]. In that work the supply chain system was modeled and optimized using mixed integer linear programming to attain the optimal utilization of marginal land for switchgrass production. The end goal for that work was to establish an economical and sustainable harvest of bioethanol. In Osmani and Zhang [49], a sustainable dual feedstock bioethanol supply chain (large-scale) was optimized in a stochastic environment. The optimization problem considered the following factors: biomass purchase and sales price; as well as biomass supply and demand. In addition to a mixed integer linear programming approach, the authors used a decomposition method: sample average approximation algorithm. Similar research efforts utilizing the mixed integer linear/nonlinear programming methodology could be observed in Osmani and Zhang [48] and Gao and You [29]. An exception to those works is seen in Marufuzzaman et al. [42] where the authors employed a combined L-shaped techniques and Lagrangian Relaxation approaches instead of a mixed integer programming methodology. In that work, insights on carbon regulatory mechanisms and other uncertainties observed in biofuel supply chains was provided. A holistic review on metaheuristic techniques implemented to bioenergy supply chains could be seen in De Meyer et al. [20] and Castillo-Villar [14].

Due to uncertain variables in biofuel supply chains, recent works have integrated fuzzy formulations into these supply chain models. A very interesting fuzzy methodology for modeling supply chains was introduced in Kozarević, S. and Puška [35]. In that work the authors proposed a method for data processing and measurement of practices and performances of supply chains. This is done by conducting applying transformation of the obtained linguistic val- ues (using appropriate fuzzy methods) into crisp values of research variable dimensions. A more practical work could be seen in Babazadeh [6]. In that work, the author developed a novel fuzzy framework for a bioenergy supply chain: the possibilistic programming model based on possibilistic mean and absolute deviation of fuzzy numbers. The model performance was evaluated by using data from a real-world case study and it was shown that the proposed method performed better than a pure possibilistic programming model. A similar work can be seen in Lin et al. [37]. In that work the uncertain factors considered were the demand of biomass energy (due to unstable price of fossil fuels) and the number of job offer opportunities springing up from the energy facilities. To account for these uncertainties the authors employed a fuzzy multiple objective linear programming to solve the problem. Another effective implementation of fuzzy framework for biofuel supply chains could be seen in the work of Balaman et al. [9]. In that work, a hybrid solution strategy combining fuzzy set theory and epsilon-constraint method was proposed. The proposed methodology was applied to handle system-specific uncertainties during the optimization of the supply chain and transportation network (entire West Midlands (WM) region of the UK). Fuzzy optimization has also been employed to model the design of renewable energy supply chains (integrated with district heating systems) (Balaman et al. [10]). In the work of Balaman et al. [10], the authors developed a novel decision model to obtain the optimal supply chain configuration and district heating system to meet the thermal demand of a certain locality. To this end, the authors formulated and validated a Fuzzy Mixed Integer Linear Programming (MILP) which consists of multiple types of biomass and systemic uncertainties.

Cuckoo search (CS) technique has been efficiently employed for optimizing real-world supply chains. A series of metaheuristics including CS was applied to a supply chain (consumerpackaged goods industry) (Mattos et al. [44]). The performance as well as the results generated by the techniques employed was presented in that work. Similar CS implementations on supply chains is given in Srivastav 
and Agrawal [58] and Abdelsalam and Elassal [1]. Supply chains models often contain many variables (large-scale) - where these variables and expressions are interlinked in a complex way. The mathematical structure (universality) of such supply chains often resemble those observed in the nuclei of heavy atoms (e.g. gold, rhodium and platinum). Random matrices were developed to specifically model complex systems which contain universality - i.e. large and complex systems with highly interconnected components (Che [16]). Random Matrix Theory (RMT) has been utilized to model such systems in:

- Solid state physics (Verbaarschot [62]; Beenakker [11])

- Quantum information theory (Collins and Nechita [18])

- Quantum chromodynamics (Akemann [4])

- Transport optimization (Krbálek and Seba [36])

- Big Data (Qiu and Antonik [52])

- Finance

It is important to note that key characteristics of supply chain networks are highly similar to those mentioned complex systems. Hence the premise: that supply chains may naturally contain universality. Following this chain of thought, the RMT framework was utilized to improve the the conventional Cuckoo Search method (CS) in this work. This was carried out by performing certain modifications to the stochastic generator component of the algorithm. In this work the conventional Gaussian stochastic generator is replaced with a RMT-based generator.

This work targets to solve the complex MO fuzzy biofuel supply chain model. The previous approaches to solve this problem uses conventional linear and nonlinear programming approaches which do not account for the complexity of the large-scale problem at hand (Ghaderi et al. [30], Chávez et al.[15], Roni et al.[54], Bairamzadeh et al.[8]). Additionally current works and tackling this problem do not consider the uncertainty in the annual electricity generation output of the biomass power plant [kWh/year]. This work contributes to the field by addressing both concerns: (1) by reformulating the MO biofuel supply chain problem as a fuzzy problem to account for uncertainties arising from the annual electricity generation output of the biomass power plant [kWh/year]. (2)The complex MO fuzzy biofuel supply chain problem is solved by using the RMT-based method - which has been observed to be very effective for solving highly complex large-scale problems (Ganesan et al. [28]).

This paper is organized as follows: Section 2 presents the fuzzy formulation of the MO biofuel supply chain model. In Section 3 the conventional CS approach is presented while an overview of RMT and its role in the development of stochastic generators is described in Section 4. Section 5 presents the results and discussion followed by the final section; conclusions and potential directions for future work.

\section{Biofuel supply chain: fuzzy formulation}

The fuel supply chain formulation utilized in this work was developed in Tan et al. [61]. In that work only two objective functions were considered: profit (Pr) and social welfare (SW). The environmental benefits objective was incorporated into the SW function. In this work, the environmental benefits function was isolated from the SW function and taken as an independent objective function (denoted Env). Various factors influence electricity generation output of biomass power plants. For instance, plant system repairs, maintenance, inspections which involve turnaround periods and downtime influence the electricity generation output. Since biomass plant type considered in this model involves various types of fuel sources (e.g. sugarcane, wheat straw, bean straw, rice husk, corncobs, branches, bark, and wood chips), the biomass plant would have to be frequently tuned to maintain robustness in the face of fuel heterogeneity. Such tuning would incur downtime which could heavily influence the power generation output. To account for these uncertain- 
ties, the fuzzy formulation was employed - where the annual electricity generation output of the biomass power plant [kWh/year] is fuzzified with its respective constraint:

$$
\sum_{t} q_{t} \in\left[Q_{\min }, Q_{\max }\right] \rightarrow \sum_{t} \tilde{q}_{t} \in\left[\tilde{Q}_{\min }, \tilde{Q}_{\max }\right]
$$

where

$$
\begin{aligned}
& \tilde{Q}_{\min }=[1260000,2340000], \\
& \tilde{Q}_{\max }=[1714000,3182000] .
\end{aligned}
$$

where the uncertainty in the annual electricity generation output of the biomass power plant is assumed to contain a variation of approximately $30 \%$ from the mean. The optimization formulation of the biofuel supply chain problem is then redefined in the fuzzy environment with the elaborated structure as follows:

Minimize (objective functions: Pr, SW, Env) subject to fuzzy constraints:

$$
\sum_{j=1}^{n} a_{i j} x_{j} \lesssim \tilde{b}_{i}, \quad i=1,2, \ldots, m
$$

and Crisp (Non-fuzzy) constraints.

The left side of $i^{\text {th }}$ fuzzy constraint in (2), $\sum_{j=1}^{n} \tilde{a}_{i j} x_{j}$ is aggregated as a fuzzy set - utilizing Zadeh's extension principle. Assuming a credibility level $\varepsilon,\left(0<\varepsilon<\frac{B}{1+C}\right)$ chosen by the decision maker, as a risk is taken and all the membership degrees smaller than $\varepsilon$ levels are ignored (Rommelfanger et al. [53]). All fuzzy data $\tilde{b_{i}} \equiv \tilde{S}\left(b_{i}^{a}, b_{i}^{b}\right)$ comprise of fuzzy variables with the following logistic membership functions (Elamvazuthi et al. [22]),

$$
\mu_{\tilde{b_{i}}}=\left\{\begin{array}{l}
1 \quad \text { if } \quad b_{i} \leqslant b_{i}^{a} \\
\frac{B \quad}{1+C e^{\alpha\left(\frac{b_{i}-b_{i}^{a}}{b_{i}^{b}-b_{i}^{a}}\right)}} \text { if } b_{i}^{a} \leqslant b_{i} \leqslant b_{i}^{b} \\
0 \quad \text { if } \quad b_{i} \geqslant b_{i}^{b}
\end{array}\right.
$$

where $\alpha=d / j$.

The fuzzy coefficients $B=1, C=0.1$ and the $\alpha \in(0,1)$. The following points are considered when we replace a crisp system by a fuzzy system (Atanu et al. [73]): (i) Specification of fuzzy inequality relations and methodology to obtain its crisp equivalents.

(ii) The interpretation 'minimization' in logistic type objective functions.

Therefore the fuzzy fuel supply chain model in this chapter consists of three objective functions to be maximized along with associated inequality constraints (see equation (9)). The objective functions are shown in equations (1)-(3):

$\operatorname{Pr}=P(1-E C) \times \sum_{t} q_{t}-$

$\left\{\begin{array}{l}F C p \\ +\sum_{t}\left[\begin{array}{l}G C \cdot q_{t} \\ +\sum_{i}\left(S C \cdot I Q_{i, t}+\sum_{k} S Q_{i, k, t} \cdot P P_{i}\right. \\ +Y_{1 t} \cdot \operatorname{extra}_{1}+Y_{2 t} \cdot \operatorname{extra}_{2}\end{array}\right]\end{array}\right]$

$$
\begin{aligned}
S W & =A C S \cdot(1-E C) \sum_{t} q_{t} \\
& +G T-G S \cdot(1-E C) \sum_{t} q_{t}
\end{aligned}
$$

$$
E n v=A C \cdot\left[\begin{array}{l}
C E T \cdot(1-E C) \cdot \sum_{t} q_{t} \\
-(C E c b-C E t p)
\end{array}\right]
$$

such that,

$$
\begin{aligned}
& C E c b=2 \sum_{i, k, t} P Q_{i, k, t} \cdot \sum_{i, k}\left[\frac{C E n c b_{i, k} \cdot D c b_{i, k}}{L C c b_{i, k}}\right] \\
& +\left(\sum_{i, k, t} P Q_{i, k, t} \cdot \sum_{i, k} C E i c b_{i, k} D c b_{i, k}\right)
\end{aligned}
$$

$$
\begin{aligned}
& C E t p=2 \sum_{i, k, t} S Q_{i, k, t} \cdot \sum_{i, k}\left[\frac{C E n t b_{i, k} \cdot D b p_{i, k}}{L C t p_{i, k}}\right] \\
& +\left(\sum_{i, k, t} P Q_{i, k, t} \cdot \sum_{i, k} C E_{i c b_{i, k}} D c b_{i, k}\right)
\end{aligned}
$$

The fuzzy constraints are as follows:

$$
\sum_{t} q_{t} \in\left[Q_{\min }, Q_{\max }\right] \rightarrow \sum_{t} \tilde{q}_{t} \in\left[\tilde{Q}_{\min }, \tilde{Q}_{\max }\right]
$$


The crisp constraints for the biofuel supply chain model are given below:

$$
\begin{gathered}
q_{t} \leqslant q_{\max } \\
I Q_{i, t} \geqslant S I l b_{i} \\
\sum_{i} I Q_{i, t} \leqslant I Q_{\max } \\
H V_{\min } \leqslant \sum_{i} H V_{i} \cdot B R_{i, t} \leqslant H V_{\max } \\
\sum_{i} S Q_{i, k, t} \geqslant S Q_{\min i, k} \\
\sum_{i} P Q_{i, k, t} \leqslant P Q_{\max } \\
\sum_{i} P Q_{i, k, t} \leqslant A Q_{\max , i, t} \\
\sum S E_{i, t}+P P_{i, k, t} \\
W E_{i, k, t} \leqslant\left[\frac{1-M C o r i_{i, t}}{1-M C_{\max . i, t}}\right]
\end{gathered}
$$

where

$$
\begin{aligned}
& E_{1}:=F C b_{k} \\
& E_{2}:=\sum_{i, t} S Q_{i, k, t} \cdot\left(\begin{array}{l}
A P_{i, k, t} \\
+\frac{T C c b_{i, k} \cdot D c b_{i k}}{L C c b_{i, k}}
\end{array}\right) \frac{1}{W R_{i, k, t}} \\
& E_{3}:=\sum_{i, t} S Q_{i, k, t} \cdot \frac{T C t p_{i, k} D b p_{k}}{L C t p_{i k}}
\end{aligned}
$$

such that,

$$
i \in[1,2], k \in[1,10], t \in[1,12]
$$

The decision parameters $q_{t}, I Q_{i, t}, S Q_{i, k, t}, P Q_{i, k, t}$ and $B R_{i, t}$. are: tails on the parameter settings of the biofuel supply chain model used in this work could be obtained in Tan et al. [61].
Tab. 1: CS Settings

\begin{tabular}{cc}
\hline Parameters & Values \\
\hline Total Number of Eggs (N) & 20 \\
Number of nests, nests & 4 \\
Lévy flight step-size, $\lambda$ & 1.5 \\
Relaxation factor, $\beta$ & 0.8 \\
Maximum & 300 \\
number iteration, $T_{\max }$ & \\
\hline
\end{tabular}

\section{Cuckoo search}

CS is a population-based stochastic search and optimization algorithm (Mareli and Twala [41]; Joshi et al. [33]). It was initially inspired by brood parasitism which was often found among certain species of cuckoo birds. This parasitism occurs when the cuckoo birds lay their eggs in the nests of other bird species (non-cuckoo birds). The heavy-tailed random walk probability distribution, Lévy flights was used as a stochastic generator for the CS technique. The iterative expression at iteration, $t$ for the candidate solution $i$ for the CS technique is:

$$
y_{i}^{t+1}=y_{i}^{t}+\beta \cdot \operatorname{Levy}(\lambda)
$$

such that the Lévy distribution is given as follows:

$$
\operatorname{Levy}(\lambda)=t^{-\lambda}
$$

wherett is the random variable, $\beta>0$ is the relaxation factor (which is modified based on the problem at hand) and $\lambda \in(1,3]$ is the Lévy flight step-size. With $t \geq 1, \lambda$ is related to the fractal dimension and the Lévy distribution becomes a specific sort of Pareto distribution. The CS algorithm is based on a few fundamental philosophies. For instance each cuckoo bird lays a single egg at one time and randomly places the egg in a selected nest. The second being: via fitness screening, the best egg (candidate solution) is carried forward into the next iteration. The worst solutions are discarded from further iterations. The nests represent the objective space (or the optimization problem landscape).

De- The parameter setting for the CS technique used in this work is shown in Tab. 1 while its respective algorithm is given in Algorithm 1: 
Algorithm 1: Cuckoo Search (CS)

Step 1: Initialize algorithmic parameters; $y_{i}, \beta$, $\lambda, N$

Step 2: Define parameters in the constraints and decision variable

Step 3: Via Lévy flights randomly lay a cuckoo egg in a nest

Step 4: Define fitness function based on solution selection criteria

Step 5: Screen eggs and evaluate candidate solution

IF: fitness criteria is satisfied

Select candidate solution (egg) to be

considered in the next iteration, $n+1$

ELSE: fitness criteria is not satisfied

Discard candidate solution (egg) from further iterations

Step 6: Rank the best solutions obtained during fitness screening

Step 7: If the fitness criterion is satisfied and $t=T_{\max }$ halt and print solutions, else proceed to Step 3.

\section{Random matrix theory \& stochastic generators}

Random Matrix Theory (RMT) is a robust mathematical framework which is very effective for describing behavior of complex systems. RMT is known to exhibit universality - a property of global symmetries shared by many systems within a certain symmetry class. Details on the application of RMT on a non-fuzzy (crisp) biofuel supply chain model could be seen in Ganesan et al. [28]. In RMT there exists two probability distributions describing: the random matrix entries and the eigenvalue spread. The nearest neighbor spacing probability distribution of eigenvalues is given by Wigner's Surmise:

$$
P(s)=A_{i} s^{i} \exp \left(-B_{i} s^{2}\right)
$$

where $s$ is the eigenvalue spacing, $A_{i}$ and $B_{i}$ are constant parameters. The normalized spacing, $s$ and the mean spacing $\langle s\rangle$ is as follows:

$$
s=\left[\frac{\lambda_{n+1}-\lambda_{n}}{\langle s\rangle}\right]
$$

such that $\langle s\rangle=\left\langle\lambda_{n+1}-\lambda_{n}\right\rangle$, where $\lambda_{n}$ is the $n^{\text {th }}$ eigenvalue sequentially such that $\lambda_{1}<\ldots<$ $\lambda_{n}<\lambda_{n+1}$. The first type of random matrices are those that are modeled based on complex quantum systems (which have chaotic classical counterparts). RMT consists of four major ensembles to determine the spacing distributions of the eigenvalues: the Gaussian Orthogonal Ensemble (GOE), Gaussian Unitary Ensemble (GUE) and Gaussian Symplectic Ensemble (GSE). In this work, the GUE distribution is considered:

$$
P(s)=\frac{32}{\pi^{2}} s^{2} \exp \left(-\frac{4}{\pi} s^{2}\right)
$$

These ensembles describe the probability density functions governing the random matrix entries. The constants, $A_{i}$ and $B_{i}$ are selected such that the following averaging properties are respected:

$$
\int_{0}^{\infty} d s P(s)=1 \text { and } \int_{0}^{\infty} d s P(s) s=1
$$

Metaheuristics are equipped with stochastic generators called the random generator - which randomly initializes the search operation of the metaheuristic. This is done by positioning the starting point of the search operation prior to exploring the objective space. In the works of Ganesan et al. [25], Ganesan et al. [26] and Ganesan et al.[27], it was seen that variations in the type of stochastic generators have an influence on the optimization results. Therefore in this work the RMT is employed as the stochastic generator to solve the fuzzy biofuel supply chain problem. Essentially RMT deals with systems with a complex network of many interlinked and interacting components - which are often encountered in real-world settings. The proposed algorithmic framework for developing a random matrix generator is as follows:

\section{Algorithm 2: Random Matrix Generator}

Step 1: Generate random eigenvalue spacings, $s$ from a GUE

Step 2: Determine the average eigenvalue spacing, $\Delta \lambda$

Step 3: Set initial eigenvalue, $\lambda_{0}$ 
Step 4: Set initial $n \times n$ matrix, $H_{i j}$

Step 5: Determine consequent eigenvalues

$$
\lambda_{i+1}=\lambda_{i}+\Delta \lambda
$$

Step 6: Determine $n \times 1$ eigenvector, $E_{i}$ :

$$
E_{i}=\sum_{j} H_{i j}+\lambda_{i}
$$

Step 7: Generate random variables from a Gaussian probability distribution function endowed eigenvector as the variance, $\sigma^{2}=E_{i}$ :

$$
P_{i}(x)=\frac{1}{\sqrt{2 \pi E_{i}^{2}}} \exp \left(-\frac{(x-\mu)^{2}}{2 E_{i}^{2}}\right)
$$

\section{Results and discussion}

The following frameworks have been introduced in the past for tackling MO optimization problems: Strength Pareto Evolutionary Algorithm (SPEA-2) (Zhao et al.[69]), Weighted sum approach (Naidu et al. [46]), Normal-Boundary Intersection (NBI) (Ahmadi et al.[2]; Ganesan et al.[23]) and Non-Dominated Sorting Genetic Algorithm (NSGA-II) (Mousavi et al.[45]). Scalarization and NBI approaches involve the aggregation of multiple target objectives. Effectively transforming the MO problem into a single objective one reduces its complexity to a high degree - making it easier to solve. In this work, the objective functions of the fuzzy MO biofuel supply chain problem was combined into a single function using the weighted sum approach (Kalita et al.[34]). This procedure effectively transforms the fuzzy MO problem into a single-objective optimization problem which can be solved for different weight values. The computational experiments employed in this work was done using the $\mathrm{C}++$ programming language on a computer using a 64-bit Win 10 platform with an Intel Core i5-7200U CPU (2.50 GHz).

Due to the stochastic nature of the algorithms employed in this work, the computational technique was executed multiple times (3 executions) and the best solution was taken. 28 solutions were obtained for a variation of weights. These

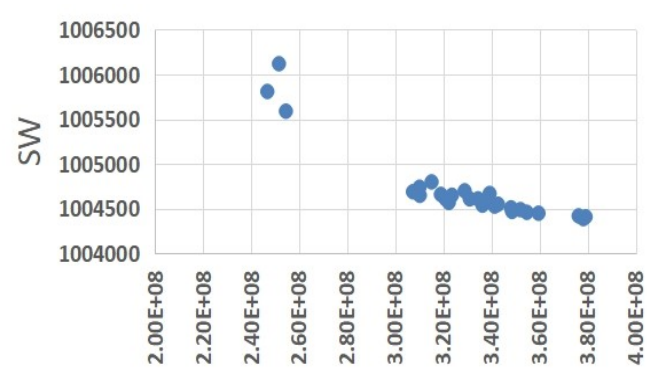

PR
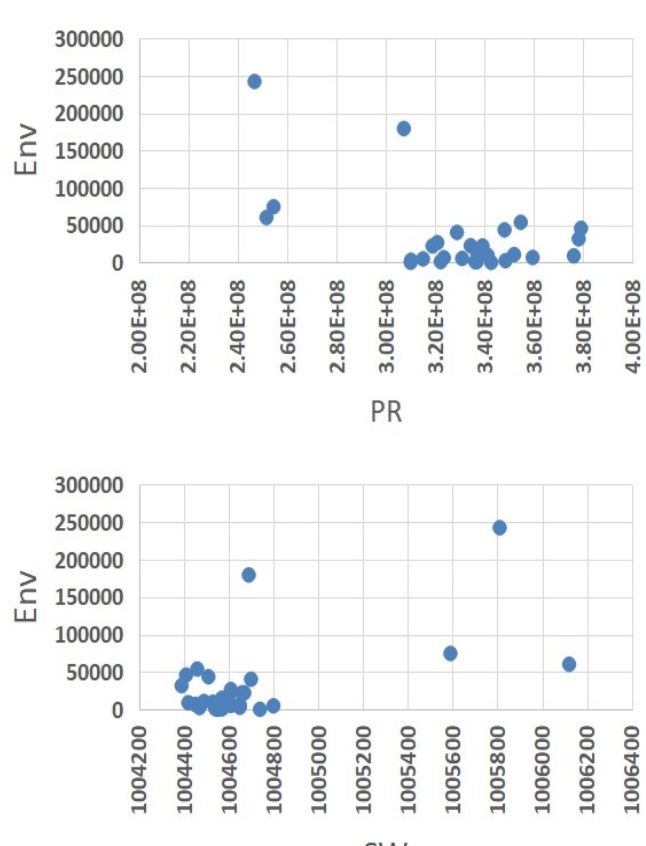

SW

Fig. 1: Pareto frontier from the fuzzy RMT-CS technique

solutions were then used to construct the Pareto frontier. - at which the individual solutions were classified as best, worst and median. The measured solutions were ranked using the hypervolume indicator (HVI) (Bringmann and Friedrich [13]). Applying the HVI, the level of solution dominance for the fuzzy MO optimization problem could be measured. A Nadir point is usually employed as a basis (or a baseline value) while measurement using the HVI. In this work, the nadir point for the HVI is computed as follows:

$$
H V I=\left[\frac{\left(z_{1}-10^{6}\right)\left(z_{2}-10^{4}\right)\left(z_{3}-10^{2}\right)}{10^{16}}\right]
$$


Tab. 2: Ranked individual solutions for the CS technique

\begin{tabular}{|c|c|c|c|c|}
\hline \multicolumn{2}{|c|}{ Description } & Best & Median & Worst \\
\hline \multirow{3}{*}{ weights } & $w_{1}$ & 0.4 & 0.2 & 0.1 \\
\cline { 2 - 5 } & $w_{2}$ & 0.2 & 0.4 & 0.3 \\
\cline { 2 - 5 } & $w_{3}$ & 0.4 & 0.4 & 0.6 \\
\hline \multirow{3}{*}{ Objective functions } & $P R$ & 246831000 & 352010000 & 342717000 \\
\cline { 2 - 5 } & $S W$ & 1005810 & 1004490 & 1004550 \\
\cline { 2 - 5 } & $E n v$ & 242631 & 10598.1 & 124.342 \\
\hline Iterations & $t$ & 100 & 78 & 67 \\
\hline Metric & $\mathrm{HVI}$ & 5937.18 & 366.46 & 0.83 \\
\hline
\end{tabular}

where $z_{1}, z_{2}$ and $z_{3}$ are individual candidate solutions. The ranked weighted individual solutions obtained using the fuzzy CS approach with random matrix generators (fuzzy RMT-CS) is given in Tab. 2. The entire Pareto frontier constructed using the fuzzy RMT-CS technique is shown in Fig. 1. In this analysis (Tab. 2 and Fig. 1), the values for the fuzzy membership function in equation (3) is fixed: $d=0.2$ and $j=0.2$.

The HVI value for the entire Pareto frontier is $27,957.73$ and the total computational time taken for construction was 33.128 seconds. To observe the variation of the objective function with respect to the membership functions, the weights are fixed to $(\mathrm{PR}, \mathrm{SW}, \mathrm{Env})=(0.4,0.2$, $0.4)$ which is the best individual solution obtained (see Tab. 2).

In Fig. 1, some prevalent trends could be observed in the distribution of the solution points in the objective space. One of these trends is the high concentration of solution points in specific regions of the objective space: $P R \in(3.0 \times$ $\left.10^{8}, 3.8 \times 10^{8}\right), S W \in(1,004,400,1,004,800)$ and Env $\in\left(0,8 \times 10^{4}\right)$. This high concentration could be attributed to the technique iteratively reaching the most optimal (local or near optimal) region of the objective space. Despite those high concentrations, some solution points could be observed to exist beyond those optimal regions. This shows that the proposed technique generates a sparse distribution of solutions and hence has good exploration capabilities - where the fuzzy RMT-CS approach explores regions in the objective space in search of other local optima.
The variation of the objective function, PR with respect to the parameters in the fuzzy membership function is given in Fig. 2:

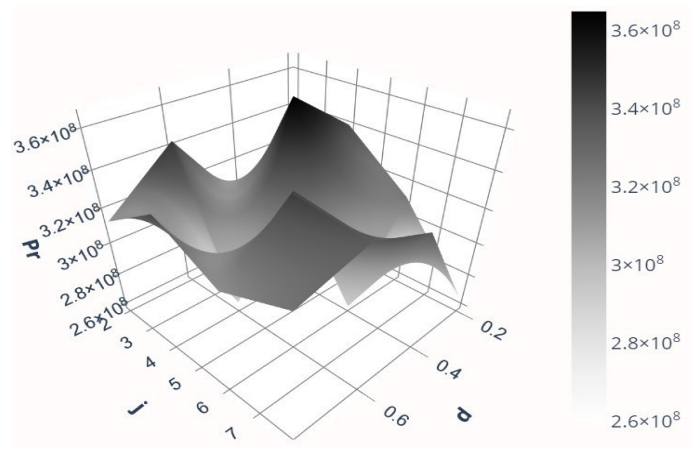

Fig. 2: Objective function, $P R$ with respect to the parameters $d$ and $j$ in the logistic membership function

In Fig. 2, the maximum value of $\mathrm{PR}$ is $365,021,000$ obtained at $d=0.4$ and $j=4$ which corresponds to $\mu=0.1$ (see equation) The median value of $\mathrm{PR}$ is $327,626,500$ and it falls between $\mu=0.05$ and $\mu=0.1$. The minimum value of $\mathrm{PR}(259,954,000)$ is obtained at $\mu=0.0667$. The objective function, SW plotted with respect to the parameters in the fuzzy membership function is given in Fig. 3:

The maximum and minimum value of the SW objective function shown in Fig. 3 were $1,005,660$ and 1,004,490 with the membership value of $\mu=0.0667$ and $\mu=0.1$. The median value obtained was $1,004,650$ which was in between $\mu=0.033$ and $\mu=0.05$. The objective function, Env with relative to the membership function is presented in Fig. 4: 


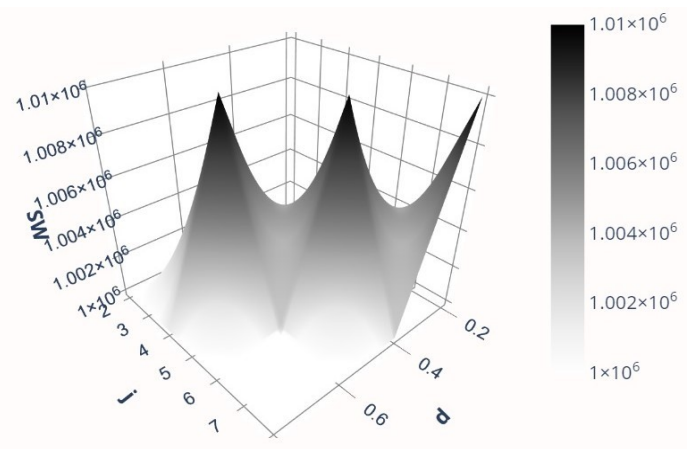

Fig. 3: Objective function, SW with respect to the parameters $d$ and $j$ in the logistic membership function

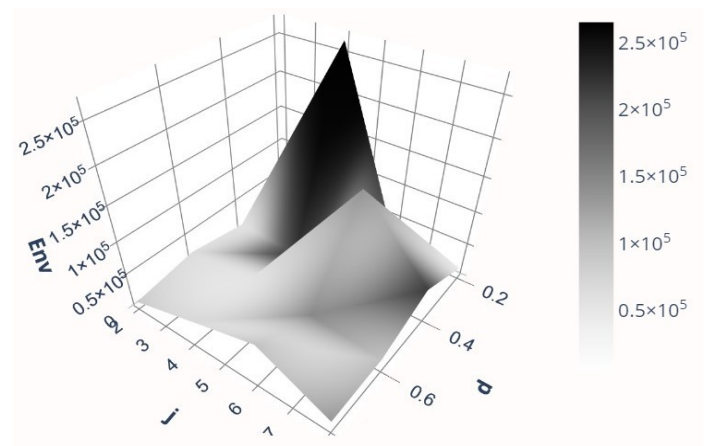

Fig. 4: Objective function, Env with respect to the parameters $d$ and $j$ in the logistic membership function

Figure 4 shows that the objective function, Env has a maximum of 266,045 at $\mu=0.05$ and minimum of $5,326.36$ at $\mu=0.1$. The median of $22,092.65$ was obtained between the membership values of $\mu=0.075$ and $\mu=0.3$.

The fuzzy RMT-CS approach produced feasible solutions; where the constraints in the fuzzy biofuel supply chain model was not broken. The computations performed during the numerical experiments were stable and the algorithm achieved convergence every time during execution. In terms of robustness, the fuzzy RMT-CS method performed stable computations and converged towards a feasible solution during each variation of the fuzzy membership function. The random matrix segment successfully complemented the CS technique to navigate through the objective space of the complex and multivariate biofuel supply chain problem.
Nevertheless the proposed technique does have some disadvantages. The first is the algorithmic complexity - where the addition of the RMT segment into the CS technique significantly increases the complexity of the algorithm. This in effect may considerably impact the computational time of the optimization process. Additionally, this technique only considers the GUE ensemble distribution and not the GOE as well as the GSE distributions for the RMT. This may adversely impact the performance of the proposed technique. Finally this work considers the uncertainty in the annual electricity generation output of the biomass power plant [kWh/year] to be of type- 1 fuzzy uncertainty. It is high possible that fluctuations in the monthly/weekly electricity generation output of the biomass power plant may more precisely capture the mentioned uncertainties - making the model more realistic.

\section{Conclusion and recommendations}

In this work, the proposed MO biofuel supply chain problem was reformulated by taking into account fluctuations in the annual electricity generation output of the biomass power plant [kWh/year]. This effectively converts the problem into a fuzzy MO problem; which is nonlinear, nonconvex and multivariate. To deal with this MO problem the CS technique was retrofitted with the RMT approach to boost its performance when faced with high levels of complexity. The proposed approach was effectively applied and Pareto efficient solutions were attained. The dominance of these solutions were gauged using the HVI.

Further computational tests could be done by using the GSE and GOE ensembles for the RMT segment in the proposed approach. In addition, RMT - based generators could also be employed to complement other metaheuristic techniques such as PSO (Mousavi et al. [45]), differential evolution (Ganesan et al. [24]) as well as other computational approaches (Ganesan et al.[25]). The accuracy of the fuzzy formulation proposed in this work could be further improved by ac- 
counting for monthly/weekly fluctuations; by reformulating the MO biofuel supply chain problem by utilizing a type-2 fuzzy framework. This work can also be extended by exploring other approaches for handling uncertainty such as: robust optimization, (Bairamzadeh et al. [7]; Kara et al.[31]), stochastic optimal control (Vinod et al. [63]) and chance constraint optimization (Cheng et al. [17]). This extension could include emerging areas of applications such as complex networks in alternative energy systems (Syahputra et al. [60]; Lotfi et al. [39]), social networks, gene networks (Youseph et al. [67]) and pharmaceutical supply chain networks (Zahiri et al. [68]).

\section{Acknowledgments}

The authors would like to sincerely thank Professor Dr. Ivan Zelinka (Technical University of Ostrava, Czech Republic) for his great help and support in this research work. This research project also supported by Modeling Evolutionary Algorithms Simulation and Artificial Intelligence (MERLIN), Faculty of Electrical \& Electronics Engineering, Ton Duc Thang University, Ho Chi Minh City, 758307, Vietnam and University Technology PETRONAS, 32610, Seri Iskandar, Malaysia.

\section{References}

[1] Abdelsalam, H. M., \& Elassal, M. M. (2014). Joint economic lot sizing problem for a three-Layer supply chain with stochastic demand. International Journal of Production Economics, 155, 272-283.

[2] Ahmadi, A., Kaymanesh, A., Siano, P., Janghorbani, M., Nezhad, A. E., \& Sarno, D. (2015). Evaluating the effectiveness of normal boundary intersection method for short-term environmental/economic hydrothermal self-scheduling. Electric Power Systems Research, 123, 192-204.

[3] Ahi, P., \& Searcy, C. (2013). A comparative literature analysis of definitions for green and sustainable supply chain management. Journal of cleaner production, 52, 329-341.

[4] Akemann, G. (2017). Random matrix theory and quantum chromodynamics. Stochastic Processes and Random Matrices: Lecture Notes of the Les Houches Summer School, 104, 228.

[5] Sengupta, A., Vasant, P., \& Andeeski, C. J. (2008). Fuzzy optimization with robust logistic membership function: A case study in for home textile industry. IFAC Proceedings Volumes, 41(2), 5262-5266.

[6] Babazadeh, R. (2019). Application of Fuzzy Optimization to Bioenergy-Supply-Chain Planning under Epistemic Uncertainty: A New Approach. Industrial \& Engineering Chemistry Research, 58(16), 6519-6536.

[7] Bairamzadeh, S., Saidi-Mehrabad, M., \& Pishvaee, M. S. (2018). Modelling different types of uncertainty in biofuel supply network design and planning: A robust optimization approach. Renewable energy, 116, 500-517.

[8] Bairamzadeh, S., Pishvaee, M. S., \& SaidiMehrabad, M. (2016). Multiobjective robust possibilistic programming approach to sustainable bioethanol supply chain design under multiple uncertainties. Industrial \& Engineering Chemistry Research, $55(1), 237-256$.

[9] Balaman, S. Y., Matopoulos, A., Wright, D. G., \& Scott, J. (2018). Integrated optimization of sustainable supply chains and transportation networks for multi technology bio-based production: A decision support system based on fuzzy $\varepsilon$-constraint method. Journal of cleaner production, 172, 2594-2617.

[10] Balaman, Ş. Y., \& Selim, H. (2016). Sustainable design of renewable energy supply chains integrated with district heating systems: A fuzzy optimization approach. Journal of cleaner production, 133, 863-885.

[11] Beenakker, C. W. J. (2015). Randommatrix theory of Majorana fermions and 
topological superconductors. Reviews of Modern Physics, 87(3), 1037.

[12] Brandenburg, M., Govindan, K., Sarkis, J., \& Seuring, S. (2014). Quantitative models for sustainable supply chain management: Developments and directions. European journal of operational research, 233(2), 299-312.

[13] Bringmann, K., \& Friedrich, T. (2013). Approximation quality of the hypervolume indicator. Artificial Intelligence, 195, 265-290.

[14] Castillo-Villar, K. K. (2014). Metaheuristic algorithms applied to bioenergy supply chain problems: theory, review, challenges, and future. Energies, 7(11), 7640-7672.

[15] Chávez, M. M. M., Sarache, W., \& Costa, Y. (2018). Towards a comprehensive model of a biofuel supply chain optimization from coffee crop residues. Transportation Research Part E: Logistics and Transportation Review, 116, 136-162.

[16] Che, Z. (2017). Universality of random matrices with correlated entries. Electronic Journal of Probability, 22.

[17] Cheng, J., Gicquel, C., \& Lisser, A. (2019). Partial sample average approximation method for chance constrained problems. Optimization Letters, 13(4), 657-672.

[18] Collins, B., \& Nechita, I. (2016). Random matrix techniques in quantum information theory. Journal of Mathematical Physics, $57(1), 015215$.

[19] Corney, J. F., \& Drummond, P. D. (2004). Gaussian quantum Monte Carlo methods for fermions and bosons. Physical review letters, 93(26), 260401.

[20] De Meyer, A., Cattrysse, D., Rasinmäki, J., \& Van Orshoven, J. (2014). Methods to optimise the design and management of biomass-for-bioenergy supply chains: A review. Renewable and sustainable energy reviews, 31, 657-670.
[21] Dong, N., Fang, X., \& Wu, A. G. (2016). A novel chaotic particle swarm optimization algorithm for parking space guidance. Mathematical Problems in Engineering, 2016.

[22] Elamvazuthi, I., Vasant, P., \& Ganesan, T. (2013). Hybrid optimization techniques for optimization in a fuzzy environment. In Handbook of Optimization (pp. 1025-1046). Springer, Berlin, Heidelberg.

[23] Ganesan, T., Vasant, P., \& Elamvazuthi, I. (2013). Normal-boundary intersection based parametric multi-objective optimization of green sand mould system. Journal of Manufacturing Systems, 32(1), 197-205.

[24] Ganesan, T., Elamvazuthi, I., \& Vasant, P. (2015). Multiobjective design optimization of a nano-CMOS voltage-controlled oscillator using game theoretic-differential evolution. Applied Soft Computing, 32, 293-299.

[25] Ganesan, T., Vasant, P., \& Elamvazuthi, I. (2016). Advances in Metaheuristics: Applications in Engineering Systems. CRC Press.

[26] Ganesan, T., Aris, M. S., \& Vasant, P. (2018). Extreme value metaheuristics for optimizing a many-objective gas turbine system. International Journal of Energy Optimization and Engineering (IJEOE), 7(2), 76-96.

[27] Ganesan, T., Aris, M. S., \& Elamvazuthi, I. (2018). Multiobjective Strategy for an Industrial Gas Turbine: Absorption Chiller System. In Handbook of Research on Emergent Applications of Optimization Algorithms (pp. 531-556). IGI Global.

[28] Ganesan, T., Vasant, P., \& Litvinchev, I. (2020). Multiobjective Optimization of a Biofuel Supply Chain Using Random Matrix Generators. In Deep Learning Techniques and Optimization Strategies in Big Data Analytics (pp. 206-232). IGI Global.

[29] Gao, J., \& You, F. (2015). Shale gas supply chain design and operations toward better economic and life cycle environmental performance: MINLP model and global 
optimization algorithm. ACS Sustainable Chemistry \& Engineering, 3(7), 1282-1291.

[30] Ghaderi, H., Moini, A., \& Pishvaee, M. S. (2018). A multi-objective robust possibilistic programming approach to sustainable switchgrass-based bioethanol supply chain network design. Journal of cleaner production, 179, 368-406.

[31] Kara, G., Özmen, A., \& Weber, G. W. (2019). Stability advances in robust portfolio optimization under parallelepiped uncertainty. Central European Journal of Operations Research, 27(1), 241-261.

[32] Hong, Y. Y., Beltran, A. A., \& Paglinawan, A. C. (2016). A chaos-enhanced particle swarm optimization with adaptive parameters and its application in maximum power point tracking. Mathematical Problems in Engineering, 2016.

[33] Joshi, A. S., Kulkarni, O., Kakandikar, G. M., \& Nandedkar, V. M. (2017). Cuckoo search optimization-a review. Materials Today: Proceedings, 4(8), 7262-7269.

[34] Kalita, K., Ragavendran, U., Ramachandran, M., \& Bhoi, A. K. (2019). Weighted sum multi-objective optimization of skew composite laminates. Structural Engineering and Mechanics, 69(1), 21-31.

[35] Kozarević, S., \& Puška, A. (2018). Use of fuzzy logic for measuring practices and performances of supply chain. Operations Research Perspectives, 5, 150-160.

[36] Krbálek, M., \& Seba, P. (2000). The statistical properties of the city transport in Cuernavaca (Mexico) and random matrix ensembles. Journal of Physics A: Mathematical and General, 33(26), L229.

[37] Lin, C. C., Liu, W. Y., \& Huang, G. L. (2019). Fuzzy Multi-objective Forest Biomass-to-biofuel Facility Location Problem with Social Consideration. Energy Procedia, 158, 4067-4072.

[38] Lin, T., Rodríguez, L. F., Shastri, Y. N., Hansen, A. C., \& Ting, K. C. (2014). Integrated strategic and tactical biomass-biofuel supply chain optimization. Bioresource technology, 156, 256-266.

[39] Lotfi, R., Mostafaeipour, A., Mardani, N., \& Mardani, S. (2018). Investigation of wind farm location planning by considering budget constraints. International Journal of Sustainable Energy, 37(8), 799-817.

[40] Luan, X. Y., Li, Z. P., \& Liu, T. Z. (2016). A novel attribute reduction algorithm based on rough set and improved artificial fish swarm algorithm. Neurocomputing, 174, 522-529.

[41] Mareli, M., \& Twala, B. (2018). An adaptive Cuckoo search algorithm for optimisation. Applied computing and informatics, 14(2), 107-115.

[42] Marufuzzaman, M., Eksioglu, S. D., \& Huang, Y. E. (2014). Two-stage stochastic programming supply chain model for biodiesel production via wastewater treatment. Computers \& Operations Research, 49, 1-17.

[43] Mastrocinque, E., Yuce, B., Lambiase, A., \& Packianather, M. S. (2013). A multiobjective optimization for supply chain network using the bees algorithm. International Journal of Engineering Business Management, 5, 38 .

[44] Mattos, C. L., Barreto, G. A., Horstkemper, D., \& Hellingrath, B. (2017). Metaheuristic optimization for automatic clustering of customer-oriented supply chain data. In 2017 12th International Workshop on Self-Organizing Maps and Learning Vector Quantization, Clustering and Data Visualization (WSOM) (pp. 1-8). IEEE.

[45] Mousavi, S. M., Sadeghi, J., Niaki, S. T. A., \& Tavana, M. (2016). A bi-objective inventory optimization model under inflation and discount using tuned Pareto-based algorithms: NSGA-II, NRGA, and MOPSO. Applied Soft Computing, 43, 57-72.

[46] Naidu, K., Mokhlis, H., \& Bakar, A. A. (2014). Multiobjective optimization using 
weighted sum artificial bee colony algorithm for load frequency control. International Journal of Electrical Power \& Energy Systems, 55, 657-667.

[47] Ogunbanwo, A., Williamson, A., Veluscek, M., Izsak, R., Kalganova, T., \& Broomhead, P. (2014). Transportation network optimization. In Encyclopedia of Business Analytics and Optimization (pp. 25702583). IGI Global.

[48] Osmani, A., \& Zhang, J. (2013). Stochastic optimization of a multi-feedstock lignocellulosic-based bioethanol supply chain under multiple uncertainties. Energy, $59,157-172$.

[49] Osmani, A., \& Zhang, J. (2014). Economic and environmental optimization of a large scale sustainable dual feedstock lignocellulosic-based bioethanol supply chain in a stochastic environment. Applied energy, 114, 572-587.

[50] Pan, I., \& Das, S. (2016). Fractional order fuzzy control of hybrid power system with renewable generation using chaotic PSO. ISA transactions, 62, 19-29.

[51] Plerou, V., Gopikrishnan, P., Rosenow, B., Amaral, L. A. N., Guhr, T., \& Stanley, H. E. (2002). Random matrix approach to cross correlations in financial data. Physical Review E, 65(6), 066126.

[52] Qiu, R. C., \& Antonik, P. (2017). Smart grid using big data analytics: a random matrix theory approach. John Wiley \& Sons.

[53] Rommelfanger, H. (1989). Interactive decision making in fuzzy linear optimization problems. European Journal of Operational Research, 41(2), 210-217.

[54] Roni, M. S., Eksioglu, S. D., Cafferty, K. G., \& Jacobson, J. J. (2017). A multiobjective, hub-and-spoke model to design and manage biofuel supply chains. Annals of Operations Research, 249(1-2), 351-380.

[55] Seuring, S. (2013). A review of modeling approaches for sustainable supply chain management. Decision support systems, 54(4), 1513-1520.
[56] Shi, J., Zhang, W., Zhang, Y., Xue, F., \& Yang, T. (2015). MPPT for PV systems based on a dormant PSO algorithm. Electric Power Systems Research, 123, 100-107.

[57] Singh, K., Vasant, P., Elamvazuthi, I., \& Kannan, R. (2015). PID tuning of servo motor using bat algorithm. Procedia computer science, 60, 1798-1808.

[58] Srivastav, A., \& Agrawal, S. (2017). Multiobjective optimization of slow moving inventory system using cuckoo search. Intelligent Automation \& Soft Computing, 1-7.

[59] Sottinen, T., \& Tudor, C. (2006). On the equivalence of multiparameter Gaussian processes.

[60] Syahputra, R., Wiyagi, R. O., Suripto, S., Jamal, A., Putra, K. T., \& Mujaahid, F. (2018). A novel fuzzy approach for multiobjective optimization of distribution network configuration in complex system. International Journal of Applied Engineering Research, 13(2), 1120-1127.

[61] Tan, Q., Wang, T., Zhang, Y., Miao, X., \& Zhu, J. (2017). Nonlinear multi-objective optimization model for a biomass directfired power generation supply chain using a case study in China. Energy, 139, 10661079.

[62] Verbaarschot, J. (2004). The supersymmetric method in random matrix theory and applications to QCD. In AIP Conference Proceedings (Vol. 744, No. 1, pp. 277-362). American Institute of Physics.

[63] Vinod, A. P., Sivaramakrishnan, V., \& Oishi, M. M. (2019). Piecewise-affine approximation-based stochastic optimal control with Gaussian joint chance constraints. In 2019 American Control Conference (ACC) (pp. 2942-2949). IEEE.

[64] Whitley, D. (2014). Sharpened and focused no free lunch and complexity theory. In Search Methodologies (pp. 451-476). Springer, Boston, MA. 
[65] Wigner, E. P. (1993). Characteristic vectors of bordered matrices with infinite dimensions i. In The Collected Works of Eugene Paul Wigner (pp. 524-540). Springer, Berlin, Heidelberg.

[66] Yang, X. S. (2013). Optimization and metaheuristic algorithms in engineering. Metaheuristics in water, geotechnical and transport engineering, 1-23.

[67] Youseph, A. S. K., Chetty, M., \& Karmakar, G. (2018). PCA based population generation for genetic network optimization. Cognitive neurodynamics, 12(4), 417429.

[68] Zahiri, B., Jula, P., \& TavakkoliMoghaddam, R. (2018). Design of a pharmaceutical supply chain network under uncertainty considering perishability and substitutability of products. Information Sciences, 423, 257-283.

[69] Zhao, F., Lei, W., Ma, W., Liu, Y., \& Zhang, C. (2016). An improved SPEA2 algorithm with adaptive selection of evolutionary operators scheme for multiobjective optimization problems. Mathematical Problems in Engineering, 2016.

[70] Bai, Z., Fang, Z., \& Liang, Y. C. (2014). Spectral theory of large dimensional random matrices and its applications to wireless communications and finance statistics: random matrix theory and its applications.

[71] Zhang, J., Osmani, A., Awudu, I., \& Gonela, V. (2013). An integrated optimization model for switchgrass-based bioethanol supply chain. Applied Energy, 102, 12051217.

[72] Zhou, C., Hou, C., Wei, X., \& Zhang, Q. (2014). Improved hybrid optimization algorithm for 3D protein structure prediction. Journal of molecular modeling, 20(7), 2289.

[73] Sengupta, A., Vasant, P., \& Andeeski, C. J. (2008). Fuzzy optimization with robust logistic membership function: A case study in for home textile industry. IFAC Proceedings Volumes, 41(2), 5262-5266.

\section{Nomenclature and abbreviations}

Biofuel Supply Chain Parameters

AC abatement cost of carbon dioxide [yuan $/ \mathrm{kg}$ ]

$\mathrm{CEicb}_{i k} \quad$ increment of carbon dioxide emissions with loading each additional ton of biomass fuel per kilometer when broker $k$ collects broker $k$ collects biomass fuel $i[\mathrm{~kg} / \mathrm{t}$ and $\mathrm{km}]$

$\operatorname{AQmax}_{i, t} \quad$ maximum available quantity of local biomass fuel $i$ in month $t$ [t/month]

ACS average electricity consumer surplus [yuan/kWh]

CEitp $_{k} \quad$ increment of carbon dioxide emissions with loading each additional ton of fuel per kilometer when broker $k$ transports biomass fuel to biomass power plant [kg/t and $\mathrm{km}]$

$\mathrm{CEncb}_{i k} \quad$ carbon dioxide emissions per kilometer when broker $k$ collects biomass fuel $I$ with no-load conveyance $[\mathrm{kg} / \mathrm{km}]$

CEntp $_{k} \quad$ carbon dioxide emissions per kilometer when broker $k$ transports biomass fuel to biomass power plant with no load conveyance $[\mathrm{kg} / \mathrm{km}]$

CET carbon dioxide emissions of thermal power plant for unit power generation $[\mathrm{kg} / \mathrm{kWh}]$ 

$\mathrm{Dcb}_{i k} \quad$ average transport distance when broker $k$ collecting biomass fuel $i[\mathrm{~km}]$
$\operatorname{Dtp}_{k} \quad$ transport distance between broker $k$ and biomass power plant $[\mathrm{km}]$
E efficiency of biomass power plant [decimal fraction]
EC electricity consumption rate of biomass power plant [decimal fraction]

extraY1 first extra cost of excessive biomass power plant fuel inventory [yuan/month]

extraY2 second extra cost of excessive biomass power plant fuel inventory [yuan/month]

ERk expected return of broker $k$ [decimal fraction mass/year]

$\mathrm{FCb}_{k} \quad$ fixed cost of broker $k$ [yuan/year]

FCp fixed cost of biomass power plant [yuan/year]

GC unit generation cost of biomass power plant [yuan $/ \mathrm{kWh}]$

GS government subsidies to biomass power generation [yuan $/ \mathrm{kWh}$ ]

GT government tax revenues from biomass power plant [yuan/year]

$\mathrm{HV}_{i} \quad$ heat value of biomass fuel $\mathrm{i}[\mathrm{kJ} / \mathrm{kg}]$

HVe heat value of electricity $[\mathrm{kJ} / \mathrm{kWh}]$
$\mathrm{HV}_{\underline{\max }}$ maximum heat value of mixed fuel $[\mathrm{kJ} / \mathrm{kg}]$

$\mathrm{HV}_{\text {min }}$ minimum heat value of mixed fuel $[\mathrm{kJ} / \mathrm{kg}]$

$\mathrm{IQ}_{i, 0} \quad$ inventory quantity of biomass fuel $i$ at the beginning of month 1 [tonnes]

CEcb carbon dioxide emissions during collecting biomass fuel $[\mathrm{kg}]$

CEtp carbon dioxide emissions during transporting biomass fuel to biomass power plant $[\mathrm{kg}]$

EIC extra inventory cost of biomass power plant [yuan]

$\mathrm{IQ}_{i, t} \quad$ inventory quantity of biomass fuel $i$ at the end of month $t$ [tonnes]

$\mathrm{PP}_{i} \quad$ purchase price of biomass fuel $i$ from brokers [yuan/t]

$\mathrm{PQ}_{i k, t} \quad$ purchase quantity of biomass fuel $i$ by broker $k$ in month $\mathrm{t}[\mathrm{t}]$

$\mathrm{q}_{t} \quad$ electricity generation of biomass power plant in month $t$ [kWh/month]

$\mathrm{R}_{t} \quad$ conversion rate from biomass fuel to electricity in month $\mathrm{t}[\mathrm{kg} / \mathrm{kWh}]$

$\mathrm{IQ}_{\max }$ maximum inventory quantity of biomass power plant $[\mathrm{t}]$

IL rate of inventory loss [decimal fraction/month]

$\mathrm{LCcb}_{i k}$ load capacity of conveyance when broker $k$ collects biomass fuel $i[\mathrm{t}]$ 
$\mathrm{LCtp}_{i k} \quad$ load capacity of conveyance

when broker $k$ transports

biomass fuel $i$ to biomass

power plant $[\mathrm{t}]$

$\operatorname{MCmax}_{i} \quad \operatorname{maximum}$ moisture content of biomass fuel $i$ required by biomass power plant [decimal fraction mass]

MCor $i i_{t}$ original moisture content of biomass fuel $i$ in month $t$ [decimal fraction mass]

MCaft $_{i k}$ moisture content of biomass fuel $i$ after processing by broker $k$

[decimal fraction mass]

$\mathrm{P} \quad$ on-grid price of biomass power plant [yuan/kWh]

$\mathrm{PQ}_{\max , k} \quad$ maximum purchasing quantity of biomass fuel by broker $k$ [t/month]

qmax maximum monthly electricity generation quantity of biomass power plant [kWh/month]

$\mathrm{Q}_{\max } \quad$ maximum annual electricity generation quantity of biomass power plant [kWh/year]

$Q_{\min } \quad$ minimum annual electricity generation of biomass power plant $[\mathrm{kWh} /$ year $]$

$\tilde{q}_{\max } \quad$ fuzzy maximum monthly electricity generation quantity of biomass power plant $[\mathrm{kWh} / \mathrm{month}]$

$\tilde{Q}_{\max } \quad$ fuzzy maximum annual electricity generation quantity of biomass power plant [kWh/year]
$\tilde{Q}_{\text {min }} \quad$ fuzzy minimum annual electricity generation of biomass power plant [kWh/year]

$R I u b_{1} \quad$ first upper bound of reasonable fuel inventory $[t]$

$R I u b_{2} \quad$ second upper bound of reasonable fuel inventory [t]

$S I l b_{i} \quad$ lower bound of safety inventory for biomass fuel i $[t]$

SC unit storage cost of biomass power plant [yuan/month]

$S Q \min _{i k}$ minimum supply quantity of biomass fuel $i$ from broker

$T C c b_{i k} \quad$ average unit transportation cost of broker $k$ when collecting biomass fuel $i$ [yuan $/ \mathrm{km}$ ]

$T C t p_{i k} \quad$ average unit transportation cost of broker $k$ when transporting biomass fuel $i$ to biomass power plant [yuan $/ \mathrm{km}$ ]

$W R_{i k, t} \quad$ ratio of the weight of biomass fuel $i$ after processing to the weight before processing by broker $k$ in month $t$ [decimal fraction mass]

$A P_{i k, t} \quad$ average price of broker $k$ buying biomass fuel $i$ in month $t$ [yuan/t] 
$B C_{i, t} \quad$ biomass fuel $i$ consumption in month $t[\mathrm{t}]$

$B R_{i, t} \quad$ blending ratio of biomass

fuel $i$ in mixed fuel

in month $t$

[decimal fraction mass]

CER carbon dioxide emissions

reduction $[\mathrm{kg}]$

$C E T_{e q}$ carbon dioxide emissions

of thermal power plant

for power generation equal

to biomass power plant $[\mathrm{kg}]$

CEB carbon dioxide emissions of biomass power plant [kg]

$S Q_{i k, t} \quad$ supply quantity of biomass

fuel $i$ by broker $k$ in month $t[\mathrm{t}]$

$V C p \quad$ total variable cost of biomass power plant [yuan/year]

$Y 1 t \quad$ binary variable to determine whether the inventory is over RIub1 at the end of month $t$

Y2t binary variable to determine whether the inventory is over RIub2 at the end of month $t$

$B, C \quad$ fuzzy coefficients

$\alpha \quad$ fuzzy membership function

$d, j \quad$ fuzzy membership values

$\varepsilon \quad$ credibility level

$a_{i j}, \tilde{b}_{i} \quad$ fuzzy constraints

PR Profits (yuan)

SW Social Welfare (yuan)

Env Environmental Benefit (kg/power station)
Random Matrix Theory and CS

$P_{1}(s) \quad$ Probabilistic Spacing

Distribution for Gaussian

Orthogonal Ensemble

(GOE)

$P_{2}(s) \quad$ Probabilistic Spacing

Distribution for Gaussian

$P_{3}(s)$

Unitary Ensemble (GUE)

Probabilistic Spacing

Distribution for Gaussian

Symplectic Ensemble

(GSE)

$P_{o}(s)$

$S$

$\lambda$

$\Delta \lambda$

$E_{i}$

$H_{i j}$

$\sigma^{2}$

$\mu$

$w_{i}$

$T_{\max }$

$m$

$H V I$

$\beta$

iter

$t$

$y^{t_{i}}$

$\operatorname{Levy}(\lambda)$

$N$

Probabilistic Spacing

Distribution for

Poisson Distribution

Eigenvalue Spacing

distribution

Eigenvalue

Eigenvalue Interval

Eigenvector

Initial Matrix

Statistical variance

Statistical Mean

Weights for the weighted sum method

Maximum limit of

function evaluations

Maximum number of

objective functions

Hypervolume Indicator

Relaxation factor

Number of algorithm iterations

Random Variable

Candidate solution

Lévy Distribution

Total Number of Eggs

\section{About Authors}

Timothy GANESAN is currently a Senior Analyst at the Royal Bank of Canada specializing in computational intelligence and data analytics. He has experience working as a Principal Researcher for the Fuels and Combustion Section in the research and development arm of the Malaysian power producer - Tenaga 
Nasional Berhad (TNB). In addition to having degrees in Chemical Engineering and Computational Fluid Dynamics, he holds a Ph.D. in Process Optimization. His research interests include engineering/industrial optimization, multi-objective/multi-level programming, evolutionary algorithms, machine learning, chaos optimization, and swarm-based optimization.

Pandian VASANT is a senior lecturer at Department of Fundamental and Applied Sciences, Faculty of Science and Information Technology, Universiti Teknologi PETRONAS in Malaysia. He holds PhD (UNEM, Costa Rica) in Computational Intelligence, MSc (UMS, Malaysia, Engineering Mathematics) and BSc (2nd Class Upper-Hons in Mathematics, UM, Malaysia) in Mathematics. His research interests include Soft Computing, Hybrid Optimization, Holistic Optimization, Innovative Computing and Applications. He has co-authored research papers and articles in national journals, international journals, conference proceedings, conference paper presentation, and special issues lead guest editor, lead guest editor for book chapters' project, conference abstracts, edited books, keynote lecture and book chapters (257 publications indexed in Web of Science). In the year 2009, Dr. Pandian Vasant was awarded top reviewer for the journal Applied Soft Computing (Elsevier), awarded outstanding reviewer in the year 2015 for ASOC (Elsevier) journal and Top reviewer for Sentinels of Science: Computer Science (Oct. 2015 - Sept. 2016). He has 26 years of working experience at the various universities from 1989-2017. Currently he is Editor-in-Chief of IJEOE and member of American Mathematical Society, ACM, SIAM, MERLIN, and NAVY Research Groups. https://publons.com/researcher/499841/drpandian-vasant-phd/

Joshua THOMAS is a senior lecturer at KDU Penang University College, Malaysia since 2008. He obtained his $\mathrm{PhD}$ (Intelligent Systems Techniques) in 2015 from University Sains Malaysia, Penang, and Master's degree in 1999 from Madurai Kamaraj University, India. From July to September 2005, he worked as a research assistant at the Artificial Intelligence
Lab in University Sains Malaysia. From March 2008 to March 2010, he worked as a research associate at the same University. Currently, he is working with Machine Learning, Big Data, Data Analytics, Deep Learning, specially targeting on Convolutional Neural Networks (CNN) and Bi-directional Recurrent Neural Networks (RNN) for image tagging with embedded natural language processing, End to end steering learning systems and GAN. His work involves experimental research with software prototypes and mathematical modelling and design $\mathrm{He}$ is an editorial board member for the Journal of Energy Optimization and Engineering (IJEOE), and invited guest editor for Journal of Visual Languages Communication (JVLC-Elsevier). He has published more than 30 papers in leading international conference proceedings and peer reviewed journals.

Igor LITVINCHEV received his M.Sc. degree in Applied Mathematics from Moscow Institute of Physics and Technology (Fizteh), Russia; Ph.D. in Systems Theory and Operations Research and Dr.Sci. (Habilitation) in Systems Modeling and Optimization from Computing Center, Russian Academy of Sciences, Moscow. He held long term visiting positions at universities in Brazil, Mexico, and Norway, as well as positions at various universities and research centers in Russia. He is an author of 4 books, editor of 5 more books and published numerous research papers focused on large-scale systems modeling, optimization, and control. His research was supported by more than 35 grants from NATO Scientic Affairs Division and European Community; ISF (USA) and RFBR (Russia); CNPq and FAPESP (Brasil); BRFBR (Belarus); CONACYT, PROMEP and PAICYT (Mexico). He is a member of Russian Academy of Natural Sciences and Mexican Academy of Sciences. 